\title{
(C) OPEN ACCESS \\ Detection of pneumothoraces in patients with multiple blunt trauma: use and limitations of eFAST
}

\author{
Thomas C Sauter, Simon Hoess, Beat Lehmann, Aristomenis K Exadaktylos, \\ Dominik G Haider
}

Department of Emergency Medicine, Inselspital, Bern University Hospital, University of Bern, Freiburgstrasse, Bern, Switzerland

\section{Correspondence to}

Thomas C Sauter, Department of Emergency Medicine, Inselspital, Bern University Hospital, Freiburgstrasse, Bern 3010, Switzerland;

Thomas.Sauter@insel.ch

Preliminary results of this study have been presented at the 28th Euroson Congress of the EFSUMB, Leipzig, Germany.

Received 18 April 2016 Revised 15 February 2017 Accepted 12 April 2017

Published Online First 12 May 2017
CrossMark

To cite: Sauter TC, Hoess S, Lehmann B, et al. Emerg Med J 2017;34:568-572.

\begin{abstract}
Background Extended focused assessment with sonography for trauma (eFAST) has been shown to have moderate sensitivity for detection of pneumothorax in trauma. Little is known about the location or size of missed pneumothoraces or clinical predictors of pneumothoraces in patients with false-negative eFAST. Methods This retrospective cross-sectional study includes all patients with multiple blunt trauma diagnosed with pneumothorax who underwent both eFAST and CT performed in the ED of a level 1 trauma centre in Switzerland between 1 June 2012 and 30 September 2014. Sensitivity of eFAST for pneumothorax was determined using CT as the gold standard. Demographic and clinical characteristics of those who had a pneumothorax detected by eFAST and those who did not were compared using the Mann-Whitney $U$ or Pearson's $\chi^{2}$ tests. Univariate binary logistic regression models were used to identify predictors for pneumothoraces in patients with negative eFAST examination.
\end{abstract}

Results The study included 109 patients. Overall sensitivity for pneumothorax on eFAST was 0.59 and 0.81 for pneumothoraces requiring treatment. Compared with those detected by eFAST, missed pneumothoraces were less likely to be ventral (30 $(47.6 \%)$ vs $4(9.3 \%), p<0.001)$ and more likely to be apical and basal $(7(11.1 \%)$ vs $15(34.9 \%), p=0.003$; $11(17.5 \%)$ vs $18(41.9 \%), p=0.008$, respectively). The missed pneumothoraces were smaller than the detected pneumothoraces (left side: $30.7 \pm 17.4$ vs $12.1 \pm 13.9 \mathrm{~mm}$; right side: $30.2 \pm 10.1$ vs $6.9 \pm 10.2 \mathrm{~mm}$, both $p<0.001$ ). No clinical variables were identified which predicted pneumothoraces in falsely negative eFAST. Among those pneumothoraces missed by eFAST, 30\% required tube thoracostomy compared with $88.9 \%$ of those detected with eFAST.

Conclusion In our study, pneumothoraces missed by eFAST were smaller and in atypical locations compared with those detected by eFAST and needed thoracic drainage less often.

\section{INTRODUCTION}

Chest trauma is responsible for approximately $20 \%$ of deaths from injury in patients with trauma. Of these $70 \%-80 \%$ are caused by road traffic accidents. Pneumothorax is a common finding, with a prevalence of up to $50 \%$ in severe chest trauma and greater than $20 \%$ in all patients with trauma. ${ }^{1}$ The triad of cardiac tamponade, tension pneumothorax and massive haemothorax in chest trauma is lethal if not rapidly recognised and treated immediately.

\section{Key messages}

What is already known on this subject?

- While there are varying estimates of sensitivity of extended focused assessment with sonography for trauma (eFAST) evaluation in patients with blunt trauma, little is known about the location or size of missed pneumothoraces. Clinical predictors for the presence of pneumothorax in patients with false-negative eFAST are also unclear.

\section{What this study adds?}

- In this retrospective study of 109 patients who received both $\mathrm{CT}$ and $\mathrm{eFAST}$, we found that pneumothoraces missed by eFAST are smaller and in atypical locations compared with those detected. Missed pneumothoraces less often needed treatment with thoracic drainage. No clinical predictors could be identified.

Pneumothoraces missed in chest radiography but detected in CT are called 'occult pneumothoraces'. The frequency of occult pneumothoraces may be up to $76 \%$ in supine CXR when read and interpreted real time by a trauma team or $2 \%-15 \%$ when later read by a board-certified radiologist. ${ }^{2}$ Although common sense might suggest that missed pneumothoraces are either very small or in atypical locations, there is no evidence to confirm this. In a comparison of CT versus CXR, the size and location of missed occult pneumothoraces in radiographic evaluation were statistically similar. ${ }^{2}$ Clinical predictors for occult pneumothoraces in CXR have been assessed but only subcutaneous emphysema and chest wall contusion have been found to be associated. ${ }^{245}$

The classic adjunct to the Advanced Traumatic Life Support (ATLS) primary survey, the focused assessment with sonography for trauma (FAST) was extended in 2004 to include a sonographic evaluation of the chest (eFAST). ${ }^{6}$ The first reviews of pneumothorax detection with sonography reported high sensitivity $(0.88$ and 0.91 , respectively), comparable to the gold standard of CT. ${ }^{78}$ In contrast to this, two recent meta-analyses found a higher rate of missed pneumothoraces (sensitivity 0.79 and 0.87 , respectively). ${ }^{9}{ }^{10}$ Sensitivity may vary greatly depending on the circumstances under which sonography was performed. A large prospective American study at a level 1 trauma centre in 2010 confirmed that single-point eFAST sonography performed by a trauma physician was both 
fast $(0.95 \mathrm{~min})$ and sensitive $(0.95) .{ }^{11}$ In contrast, a similar study in 2015 concluded that the sensitivity of eFAST in patients with blunt chest trauma was only $0.42 .^{12}$

There have been few studies of the location or size of missed pneumothoraces in the eFAST examination of patients with multiple blunt trauma. This study seeks to describe the characteristics of patients with missed pneumothorax in eFAST and identify clinical or patient characteristics that might predict pneumothoraces in patients with missed pneumothoraces in eFAST in general and specifically in the subgroup of pneumothoraces that needed treatment with thoracic drainage.

\section{MATERIALS, METHODS AND PATIENTS}

This retrospective cross-sectional study includes all patients directly admitted to the ED of the University Bern Hospital Inselspital, Bern, Switzerland, between 1 June 2012 and 30 September 2014 who were diagnosed with pneumothorax after blunt trauma and underwent both an eFAST and a chest CT. The ED is a level 1 trauma centre, treats about 40000 patients per year and has a catchment area of 2 million people. The ED is a self-contained, interdisciplinary unit treating approximately 500 multi-injured patients (Injury Severity Index $>16$ ) per year.

During the study period, electronic data were recorded in the computerised database (ED 2.1.3.0; E-Care, Turnhout, Belgium). Medical records were electronically screened for the search term 'pneumothorax'. Patients with a documented diagnosis of pneumothorax were further evaluated. Patients without both eFAST and CT completed were excluded. In order to minimise selection bias, we furthermore excluded patients secondarily transferred to our level 1 hospital with the diagnosis of pneumothorax, patients with non-traumatic pneumothorax as well as penetrating trauma (figure 1). Demographic data (age, gender, nationality) were collected as well as clinical data on admission to the ED (mechanism of blunt trauma, haemodynamic instability at admission (defined as positive shock index (HR > systolic BP)), GCS, weight, oxygen saturation at admission, subcutaneous emphysema, administration of oxygen at admission, invasive ventilation), imaging modalities and results (side, location and extension of pneumothorax) and treatment characteristics (thoracic drainage, surgical treatment after initial management in the trauma room (referral to theatre)).

CT is the gold standard for detection of pneumothorax and was used for comparison. All CT scans were read by board-certified radiologists. The size of the pneumothorax was assessed by its depth (distance of pleural line from chest wall) on the CT. All sonographic examinations were done on a HI VISION Avius (Hitachi Aloka Medical Systems) with a curved array probe (EUP-C715, $5 \mathrm{MHz}$; Hitachi Aloka Medical Systems). The curved array probe is used for the whole abdominal and thoracic eFAST evaluation in order to shorten examination times.

The eFAST investigation was performed during the primary survey in the trauma room by consultants in emergency medicine with training in sonography. With respect to investigation time, scanning location and sequence of investigation, we adhered to the recommendations for eFAST in the trauma room by Kirkpatrick et al. ${ }^{6}$ Bilateral (two-point) longitudinal parasternal views were obtained to rule out pneumothorax. Evidence for pneumothorax includes the absence of gliding of the visceral pleura against the parietal pleura, the 'stratosphere or barcode sign' visible in the M-mode, the absence of a lung pulse and the absence of comet tail artefacts (a type of

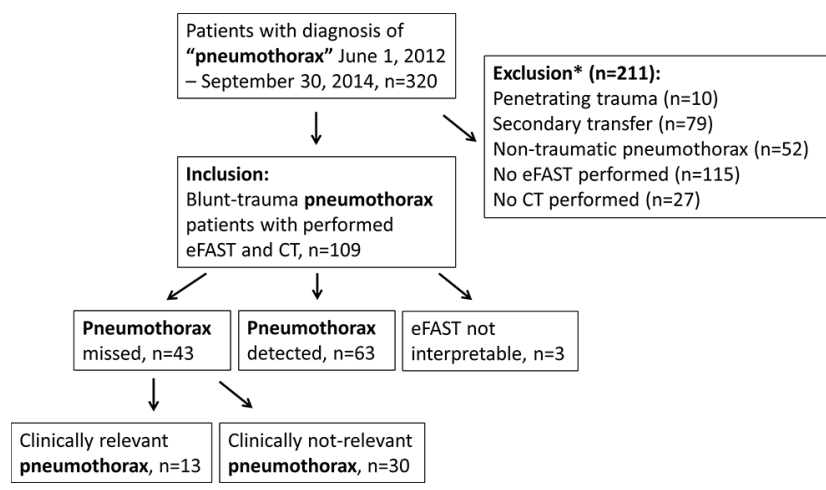

Figure 1 Flowchart. *More than one exclusion may apply to each excluded patient.

reverberation artefact first described by Ziskin et al). ${ }^{13-15}$ The eFAST investigation during the primary survey was intended to take less than $2 \mathrm{~min}$.

The study protocol was approved and registered by the Ethics Committee of Canton Bern, Switzerland (number 155/2015). No informed consent was necessary according to the Ethics Committee because of the retrospective study design and the handling of anonymised data.

Descriptive statistics were used when applicable. For group comparisons, independent samples t-tests or Mann-Whitney U tests (normally distributed data) or Pearson's $\chi^{2}$ tests or Fisher's exact test (categorical variables) were used as appropriate. To identify associations between clinical predictors and pneumothoraces missed in eFAST, we used univariate binary logistic regression models. The convenience sample for this study was obtained from the computerised database of the patients admitted to our ED. The studies power to detect a difference in size of pneumothorax of $20 \mathrm{~mm}$ with an alpha of 0.05 is $99.9 \%$ given the sample size, means and SD observed in this study.

The SPSS Statistics programme (IBM) was used for all statistical calculations and a two-tailed $p$ value of $<0.05$ was considered statistically significant.

\section{RESULTS}

In total, 320 patients with documented pneumothorax could be identified from 1 June 2012 to 30 September 2014 (figure 1). After exclusion of 211 patients due to penetrating trauma $(n=10)$, secondary transfer $(n=79)$ or incomplete imaging $(n=142), 109$ patients with pneumothorax after blunt trauma and eFAST as well as CT imaging were included in our study. In three patients, the eFAST examination was attempted but not interpretable for organisational or technical reasons. These patients were therefore excluded from further analysis.

The final 106 patients had a mean age of $48.6 \pm 19.3$ years and were predominantly male $(n=82,77.4 \%)$. Falls were the most common type of trauma $(n=50,47.2 \%)$ followed by motorbike and car accidents $(n=39,36.8 \%)$; table 1$)$.

Sixty-three pneumothoraces were detected by eFAST out of 106 pneumothoraces with an overall sensitivity for the detection of pneumothoraces in eFAST of 0.59 . In the subgroup of pneumothoraces that needed treatment with thoracic drainage, the sensitivity of eFAST was 0.81 . The group comparison of patients with detected or missed pneumothoraces in eFAST is shown in table 1 . 
Table 1 Group comparison of patient groups with detected or missed PTX in eFAST examination

\begin{tabular}{|c|c|c|c|c|}
\hline Parameter & $\begin{array}{l}\text { PTX total } \\
(\mathrm{N}=106)\end{array}$ & $\begin{array}{l}\text { PTX detected } \\
(n=63)\end{array}$ & $\begin{array}{l}\text { PTX missed } \\
(n=43)\end{array}$ & p Value \\
\hline Age (years) & $48.6 \pm 19.3$ & $51.6 \pm 18.5$ & $44.3 \pm 19.8$ & 0.053 \\
\hline Gender (male) & $82(77.4 \%)$ & $49(77.8 \%)$ & $33(76.7 \%)$ & 0.901 \\
\hline Weight $(\mathrm{kg})$ & $81.6 \pm 14.4$ & $84.4 \pm 14.9$ & $76.8 \pm 12.8$ & 0.132 \\
\hline \multicolumn{5}{|l|}{ Type of trauma } \\
\hline Motorbike/car accident & $39(36.8 \%)$ & $23(36.5 \%)$ & $16(37.2 \%)$ & 0.941 \\
\hline Bicycle accident & $9(8.5 \%)$ & $4(6.3 \%)$ & $5(11.6 \%)$ & 0.481 \\
\hline Fall & $50(47.2 \%)$ & $29(46.0 \%)$ & $21(48.8 \%)$ & 0.776 \\
\hline Blunt direct trauma & $8(7.5 \%)$ & $7(11.1 \%)$ & $1(2.3 \%)$ & 0.138 \\
\hline $\begin{array}{l}\text { Side of PTX } \\
\text { (left right; bilateral) }\end{array}$ & $\begin{array}{l}44(41.5 \%) ; 51(48.1) ; \\
11(10.4 \%)\end{array}$ & $\begin{array}{l}26(41.3 \%) ; 30(47.6 \%) \\
7(11.1 \%)\end{array}$ & $\begin{array}{l}18(41.9 \%) ; 21(48.8 \%) ; \\
4(9.3 \%)\end{array}$ & $\begin{array}{l}0.684 ; 0.873 \\
0.803\end{array}$ \\
\hline \multicolumn{5}{|l|}{ Location* } \\
\hline Ventral & $37(34.9 \%)$ & $33(52.4 \%)^{*}$ & $4(9.3 \%)^{*}$ & $<0.001$ \\
\hline Apical & $24(22.6 \%)$ & $7(11.1 \%)^{*}$ & $17(39.5 \%)^{*}$ & 0.001 \\
\hline Basal & $31(29.2 \%)$ & $12(19.0 \%)^{*}$ & $19(44.2 \%)^{*}$ & 0.005 \\
\hline Lateral & $19(17.9 \%)$ & $14(22.2 \%)$ & $5(11.6 \%)$ & 0.163 \\
\hline Size PTX (left; right) (mm) & $23.2 \pm 18.4 ; 20.9 \pm 19.8$ & $30.7 \pm 17.4^{*} ; 30.2 \pm 19.1^{*}$ & $12.1 \pm 13.9 * ; 6.9 \pm 10.2 *$ & $<0.001 ;<0.001$ \\
\hline \multicolumn{5}{|l|}{ Medical data } \\
\hline Thorax drainage & $69 \%(65.1 \%)$ & $56(88.9 \%)^{*}$ & $13(30.2 \%)^{*}$ & $<0.001$ \\
\hline Subcutaneous emphysema & $20(18.9 \%)$ & $15(23.8 \%)$ & $5(11.6 \%)$ & 0.115 \\
\hline Invasive ventilation & $20(18.9 \%)$ & $14(22.2 \%)$ & $6(14.0 \%)$ & 0.292 \\
\hline Unstable at admission & $15(14.2 \%)$ & $10(15.9 \%)$ & $5(11.6 \%)$ & 0.496 \\
\hline Oxygen administered at admission & $94(88.7 \%)$ & $56(88.9 \%)$ & $38(88.4 \%)$ & 0.644 \\
\hline Oxygen saturation at admission (\%) & $97.3 \pm 4.6$ & $97.8 \pm 4.7$ & $96.9 \pm 4.6$ & 0.306 \\
\hline GCS at admission & $12.3 \pm 4.5$ & $12.3 \pm 4.5$ & $12.4 \pm 4.4$ & 0.865 \\
\hline Surgical intervention & $41(38.7 \%)$ & $21(33.3 \%)$ & $20(46.5 \%)$ & 0.166 \\
\hline
\end{tabular}

* Multiple locations for each single patient are possible.

Values with statistical significance are in bold.

Mean \pm SD or absolute numbers (\%), independent samples t-test, Mann-Whitney U, Pearson's $\chi^{2}$ or Fisher's exact tests as applicable, * $p<0.05$, N=106.

eFAST, extended focused assessment with sonography for trauma; PTX, pneumothorax.

There were no differences in gender, age, weight or type of trauma between patients with missed or detected pneumothorax by eFAST.

The side of the pneumothorax was not significantly different in the two groups (left: $26(41.3 \%)$ vs 18 (41.9\%); right: 30 $(47.6 \%)$ vs $21(48.8 \%), p=0.684$ and $\mathrm{p}=0.873)$. Compared with detected pneumothoraces, missed pneumothorax were significantly less likely to be ventral in location (33 (52.4\%) vs $4(9.3 \%), \mathrm{p}<0.001)$ but significantly more likely to be apical and basal (7 (11.1\%) vs $17(39.5 \%), \mathrm{p}=0.001 ; 12(19.0 \%)$ vs $19(44.2 \%), p=0.005$, respectively). About half of the detected pneumothoraces were found in the ventral location (52.4\%), but more than two-thirds of missed pneumothoraces were found to be apical or basal $(83.7 \%)$.

The pneumothoraces missed in the eFAST examination were smaller on both sides (left side: $30.7 \pm 17.4$ vs $12.1 \pm 13.9 \mathrm{~mm}$; right side: $30.2 \pm 10.1$ vs $6.9 \pm 10.2 \mathrm{~mm}$, both $\mathrm{p}<0.001)$.

Pneumothoraces detected in the eFAST examination needed treatment in $88.9 \%$ of cases. In contrast to this, pneumothoraces missed in eFAST needed treatment in $30.2 \%$ of cases $(\mathrm{p}<0.001)$. The cases of patients who were unstable at admission $(n=5)$ and with missed pneumothorax in eFAST were specifically reviewed. These pneumothoraces were apical $(n=2,40 \%)$, basal $(n=2$, $40 \%)$ and lateral $(n=1,20 \%)$. The mean size of these pneumothoraces was $6.4 \pm 7.7 \mathrm{~mm}$ (range $2-20 \mathrm{~mm}$ ). In all of these cases, the CT as read by a board-certified radiologist showed no signs of a tension pneumothorax.
No other tested parameter, including subcutaneous emphysema and weight, was associated with pneumothoraces in patients with negative eFAST (table 2).

Table 2 Associations of missed pneumothorax in eFAST with clinical and patient characteristics in univariate analysis

\begin{tabular}{lll}
\hline Parameter & $\mathrm{p}$ Value & OR (Cl) \\
\hline Age (years) & 0.056 & $0.980(0.959$ to 1.001$)$ \\
Gender & 0.901 & $0.943(0.374$ to 2.375$)$ \\
Nationality & 0.413 & $1.608(0.516$ to 5.011$)$ \\
\hline Weight & 0.138 & $0.957(0.902$ to 1.014$)$ \\
Direct trauma & 0.128 & $0.190(0.023$ to 1.608$)$ \\
\hline Fall & 0.776 & $1.119(0.515$ to 2.433$)$ \\
\hline Bicycle accident & 0.345 & $1.941(0.490$ to 7.688$)$ \\
\hline Car accident & 0.941 & $1.031(0.462$ to 2.301$)$ \\
Emphysema & 0.123 & $0.421(0.140$ to 1.262$)$ \\
\hline Unstable at admission & 0.498 & $0.671(0.212$ to 2.125$)$ \\
Oxygen saturation at & 0.311 & $1.052(0.954$ to 1.159$)$ \\
admission (\%) & & \\
GCS at admission & 0.907 & $1.005(0.921$ to 1.098$)$ \\
Oxygen administered at & 0.646 & $0.679(0.130$ to 3.542$)$ \\
admission & & \\
\hline Invasive ventilation & 0.296 & $0.571(0.200$ to 1.632$)$ \\
Surgical intervention & 0.168 & $1.769(0.787$ to 3.977$)$ \\
\hline
\end{tabular}

Binary logistic regression, ${ }^{*} p<0.05$.

eFAST, extended focused assessment with sonography for trauma. 


\section{DISCUSSION}

Our study showed that missed pneumothoraces were significantly smaller and in more atypical locations than pneumothoraces detected in eFAST. The patients with missed pneumothorax less frequently needed thoracic drainage. Instability did not appear to be associated with a missed pneumothorax. There were no differences in gender, nationality or age, and no type of trauma was specifically prone to failure to detect a pneumothorax on EFAST.

\section{Sensitivity of eFAST in our study population}

The overall sensitivity for detection of pneumothoraces with eFAST in our study was just below the findings of previous studies. ${ }^{9} 10$ In all sonographical examinations, the results largely depend on the experience of the operators and the environment in which the examination is done. Another factor that may cause the overall sensitivity in our study to be below the results previously quoted in the literature is that in order to compare eFAST with CT, patients without completed CT were excluded. Therefore, large pneumothoraces which need immediate thoracostomy after eFAST but before CT imaging are not represented in these results. It is, however, reassuring that in the subgroup of patients with pneumothorax which needed thoracic drainage and were therefore considered clinically relevant in the acute setting, the sensitivity was found to be 0.81 , which was much higher than the overall sensitivity. This sensitivity is in the upper range of the reported values. ${ }^{9} 10$

\section{What are the characteristics of patients with missed pneumothorax in eFAST?}

The pneumothoraces missed in this study were significantly smaller and needed significantly fewer treatments than the detected pneumothoraces. The detected pneumothoraces were mainly found in the expected typical location (ventral), in contrast to the missed pneumothoraces that were mainly in an atypical location (basal or apical). In view of the focused two-point eFAST examination technique used, it is not surprising that small pneumothoraces in the upper and lower parts of the thorax were missed. This is in contrast to studies which compared CXR with CT and found no particular distribution of missed pneumothoraces. ${ }^{3}{ }^{4}$ It might be valuable to discuss extending the eFAST examination to cover more locations on both sides of the thorax. On the other hand, no pneumothorax was missed that caused instability, so that the established procedure of a two-point examination seems a reasonable compromise between examination speed and accuracy.

\section{Is it possible to identify clinical characteristics that might predict pneumothoraces in patients with negative eFAST in general or in the subgroup of pneumothoraces that needed treatment with thoracic drainage?}

Previous studies of predictors of occult pneumothoraces in CXR remain rather unsatisfying. The only identified predictors for occult pneumothoraces in CXR are subcutaneous emphysema and chest wall contusion. ${ }^{245}$ This difficulty seems to be the same with eFAST. Subcutaneous emphysema failed to predict missed pneumothorax in eFAST. We could not test 'chest wall contusion', the other predictor, because this was not reliably recorded on a regular basis in our medical records. Although increased patient weight might be suspected to make an eFAST examination more difficult, weight was not associated with the detection of pneumothorax. No patient factors or mechanisms of trauma were associated with prediction.

\section{Limitations}

Because these data were extracted retrospectively from medical records not collected by the authors, as in all retrospective research, there is no guarantee of the completeness and correctness of the recorded patient data. As the eFAST examinations in our study were performed by a physician in a real-life trauma room setting, interobserver and intraobserver variability cannot be excluded. All physicians performing the ultrasound were specifically trained in eFAST and take part in mandatory ultrasound refresher training. An independent review of ultrasound images could reduce this potential bias and should be conducted in further investigations.

Decisions about imaging modalities and treatment were made by the treating physician. Because the reasons for these decisions were not explicitly documented, we have not speculated further to avoid misleading conclusions.

Due to the small sample size, type II error cannot be excluded for comparisons between the patients with detected and missed pneumothoraces. To overcome these potential biases, larger prospective multicentre investigations should be performed. Another potential limitation may be that a pneumothorax could develop over time. Although time between eFAST and CT is minimised, the progression or development of a pneumothorax during the time between the eFAST and CT in this investigation cannot be completely excluded.

\section{CONCLUSION}

This study demonstrates that missed pneumothoraces in eFAST are significantly smaller and are in more atypical locations than the detected pneumothoraces. Missed pneumothoraces were less likely to need thoracic drainage. As a missed pneumothorax was not linked with haemodynamical instability in any patient, it may be concluded that the focused two-point eFAST examination in patients with blunt trauma is adequate. However, larger studies are needed.

Contributors All authors significantly contributed to the final manuscript in its present form, including conception and design of the study, drafting of the manuscript and final approval of the manuscript.

Competing interests None declared.

Patient consent No informed consent is necessary according to the Ethics Committee, because of the retrospective design and the handling of anonymised data.

Ethics approval The study protocol was approved and registered by the Ethics Committee of Canton Bern, Switzerland (number 155/2015).

Provenance and peer review Not commissioned; externally peer reviewed.

Data sharing statement All presented data can be obtained from the corresponding author.

Open Access This is an Open Access article distributed in accordance with the Creative Commons Attribution Non Commercial (CC BY-NC 4.0) license, which permits others to distribute, remix, adapt, build upon this work non-commercially, and license their derivative works on different terms, provided the original work is properly cited and the use is non-commercial. See: http://creativecommons.org/ licenses/by-nc/4.0/

(c) Article author(s) (or their employer(s) unless otherwise stated in the text of the article) 2017. All rights reserved. No commercial use is permitted unless otherwise expressly granted.

\section{REFERENCES}

1 Di Bartolomeo S, Sanson G, Nardi G, et al. A population-based study on pneumothorax in severely traumatized patients. J Trauma 2001;51:677-82. 


\section{Original article}

2 Ball CG, Kirkpatrick AW, Feliciano DV. The occult pneumothorax: what have we learned? Can J Surg 2009;52:E173-9.

3 Ball CG, Kirkpatrick AW, Laupland KB, et al. Factors related to the failure of radiographic recognition of occult posttraumatic pneumothoraces. Am J Surg 2005;189:541-6.

4 Misthos P, Kakaris S, Sepsas E, et al. A prospective analysis of occult pneumothorax delayed pneumothorax and delayed hemothorax after minor blunt thoracic trauma. Eur J Cardiothorac Surg 2004;25:859-64.

5 Ball CG, Ranson K, Dente CJ, et al. Clinical predictors of occult pneumothoraces in severely injured blunt polytrauma patients: a prospective observational study. Injury 2009;40:44-7.

6 Kirkpatrick AW, Sirois M, Laupland KB, et al. Hand-held thoracic sonography for detecting post-traumatic pneumothoraces: the Extended Focused Assessment with Sonography for Trauma (EFAST). J Trauma 2004;57:288-95.

7 Ding W, Shen Y, Yang J, et al. Diagnosis of pneumothorax by radiography and ultrasonography: a meta-analysis. Chest 2011;140:859-66.

8 Alrajhi K, Woo MY, Vaillancourt C. Test characteristics of ultrasonography for the detection of pneumothorax: a systematic review and meta-analysis. Chest 2012:141:703-8.
9 Alrajab S, Youssef AM, Akkus NI, et al. Pleural ultrasonography versus chest radiography for the diagnosis of pneumothorax: review of the literature and metaanalysis. Crit Care 2013;17:R208.

10 Ebrahimi A, Yousefifard M, Mohammad Kazemi H, et al. Diagnostic accuracy of chest ultrasonography versus chest radiography for identification of pneumothorax: a systematic review and meta-analysis. Tanaffos 2014;13:29-40.

11 Nandipati KC, Allamaneni S, Kakarla R, et al. Extended Focused Assessment with Sonography for Trauma (EFAST) in the diagnosis of pneumothorax: experience at a community based level I trauma center. Injury 2011:42:511-4.

12 Abdulrahman Y, Musthafa S, Hakim SY, et al. Utility of extended FAST in blunt chest trauma: is it the time to be used in the ATLS algorithm? World I Surg 2015;39:172-8.

13 Ziskin MC, Thickman DI, Goldenberg NJ, et al. The comet tail artifact. J Ultrasound Med 1982;1:1-7.

14 Lichtenstein DA, Menu Y. A bedside ultrasound sign ruling out pneumothorax in the critically ill. lung sliding. Chest 1995;108:1345-8.

15 Lichtenstein D, Mezière G, Biderman P, et al. The comet-tail artifact: an ultrasound sign ruling out pneumothorax. Intensive Care Med 1999:25:383-8. 